Authors

Robert K. Shelton, Long-Sheng Ma, Henry C. Kapteyn, Margaret M. Murnane, John L. Hall, and Jun Ye 


\title{
Phase-Coherent Optical Pulse Synthesis from Separate Femtosecond Lasers
}

\author{
Robert K. Shelton, ${ }^{*}$ Long-Sheng Ma, ${ }^{*} \dagger$ Henry C. Kapteyn, \\ Margaret M. Murnane, John L. Hall, Jun Ye*
}

\begin{abstract}
We generated a coherently synthesized optical pulse from two independent mode-locked femtosecond lasers, providing a route to extend the coherent bandwidth available for ultrafast science. The two separate lasers (one centered at 760 nanometers wavelength, the other at 810 nanometers) are tightly synchronized and phase-locked. Coherence between the two lasers is demonstrated via spectral interferometry and second-order field cross-correlation. Measurements reveal a coherently synthesized pulse that has a temporally narrower second-order autocorrelation width and that exhibits a larger amplitude than the individual laser outputs. This work represents a new and flexible approach to the synthesis of coherent light.
\end{abstract}

The interaction of coherent light with matter, and the "control" of matter using light, has been a prominent scientific theme in recent years. On "slow" time scales, the interaction of precisely tuned continuous wave $(\mathrm{CW})$ laser light with atomic vapors leads to the generation of quantum degenerate gases (1) and deterministic quantum entanglement among atoms (2). On fast time scales, ultrashort light pulses are used to study coherent evolution in atomic and molecular systems. "Coherent control" experiments now use light pulses, precisely shaped in amplitude and phase, to selectively "drive" (3) a desired dynamical process.

The ability to generate coherent light with ultrabroad bandwidths is essential for many applications in ultrafast science and technology. Advances in this area have led to broadbandwidth, ultrashort-pulse, laser systems (4) that routinely generate pulses shorter than 10 femtoseconds (fs) $(5,6)$. Moreover, coherent bandwidths can be extended over very broad spectral regions by using nonlinear frequency conversion techniques such as white-light continuum generation $(7,8)$, parametric amplification (9), molecular phase modulation (10), and so forth. However, for some applications, these techniques can suffer from poor efficiency and lack of flexibility, in particular for applications where arbitrary pulse synthesis at very different wavelengths is required. Ideally, it would be desirable to

JILA (formerly Joint Institute for Laboratory Astrophysics), National Institute of Standards and Technology (NIST) and University of Colorado, Department of Physics, University of Colorado, Boulder, CO 80309-0440, USA.

*These authors contributed equally to this work. $\dagger$ Permanent address: Physics Department, East China Normal University, Shanghai, China.

\$To whom correspondence should be addressed. Email: ye@jila.colorado.edu synthesize arbitrary pulses by coherently combining the output fields of two or more separate lasers, which would enable the generation of optical waveforms with distinct optical properties in distinct regions of the spectrum with potentially high powers. Here, we report the demonstration of coherent optical pulse synthesis from two independent fs lasers. This approach will have application in many areas of science and technology, including wide-bandwidth pump-probe configurations and coherent control, mid-infrared generation through difference frequency mixing (11), laser synchronization with $\mathrm{x}$-rays or electron beams from synchrotrons (12), particle acceleration with phase-locked pulsed laser arrays (13), and synthesis of light pulses with durations shorter than those obtainable from any individual laser (4-6).

Tight phase locking of two CW lasers is a mature field (14) and has been applied to many scientific experiments. Phase locking of separate fs lasers, however, requires both tight synchronization between the two pulse trains as well as effective detection of the phase difference between the two optical carrier waves underlying the pulse envelopes. Recently, the combination of ultrafast laser technology with precision frequency metrology based on CW lasers (15) has resulted in dramatic breakthroughs of significance to both fields. Optical frequencies can now be directly linked to microwave sources (16, 17), and the stability of a CW optical oscillator can be readily transferred to the entire optical frequency comb $(18,19)$. In the time domain, control over mode frequencies translates into control over the time domain evolution of the carrier-envelope phase of the short pulses $(20,21)$. Here, we take advantage of these concepts of control in both the time and frequency domain to demonstrate coherent light synthesis: the coherent combi- nation of outputs from more than one laser in such a way that the combined output can be viewed as a coherent, fs-duration pulse that is emitted from a single source.

As a first step, we synchronize two Kerrlens mode-locked (KLM) fs lasers. The KLM laser generates a repetitive pulse train, and the rigorous periodicity in time leads to a corresponding periodicity in the spectral domain. The repetition rates of the two lasers are required to be identical to a very high degree of precision. The repetition rate of each laser has been controlled precisely using either a microwave clock (16) or a stable reference laser $(18,19)$. Tight synchronization between the two separate lasers is achieved via direct phase locking of the repetition frequencies of the two lasers.

However, in order to phase lock the optical carrier frequencies of the two lasers, it is necessary to ensure that the spectral combs of the individual lasers are exactly coincident in the region of spectral overlap. This requirement is associated with the rate of slipping of the carrier-envelope phase of these short pulses. The difference in the group $\left(v_{\mathrm{g}}\right)$ and phase $\left(v_{\mathrm{p}}\right)$ velocities $\left(v_{\mathrm{p}} \neq v_{\mathrm{g}}\right)$ results in a pulse envelope function that is not fixed with respect to the underlying optical oscillation frequencies - in general, there is a phase slip between the "carrier" phase and the envelope peak for each of the successive pulses emitted by the laser. Denoting this pulse-to-pulse phase slip by $\Delta \phi$, each optical comb frequency will be given by $f_{\mathrm{n}}=n f_{\text {rep }}-\delta$, which corresponds to $\delta=f_{\text {rep }}(\Delta \phi / 2 \pi)$, where $n$ is the integer harmonic number $\left(\sim 4 \times 10^{6}\right)$ of the optical comb line relative to $f_{\text {rep }}$. Thus, while the comb lines are rigorously equally spaced in frequency, they are not necessarily harmonics of the repetition rate $f_{\text {rep}}$; i.e., extending the comb toward zero frequency would reveal a frequency offset of the closest comb line from zero frequency (22).

To clarify the situation further, the fundamental issues of phase locking two modelocked lasers are illustrated in both the time domain (Fig. 1A) and frequency domain (Fig. 1B). The two pulses need to be synchronized tightly, i.e., the two repetition rates are controlled to match each other $\left(f_{\text {rep } 1}=f_{\text {rep } 2}\right)$. To achieve phase locking under synchronization, we also need to match the two sets of optical frequency combs so that they form a continuous and phased set. To achieve this, the two lasers should exhibit a certain degree of spectral overlap (Fig. 1B), allowing detection of a coherent heterodyne beat signal between the corresponding comb components of the two lasers. Such heterodyne detection yields information related to $\left(\delta_{1}-\delta_{2}\right)$, which can then be controlled. By phase locking $\left(\delta_{1}\right.$ $-\delta_{2}$ ) to a frequency of a mean zero value, we effectively demand that $\left(\Delta \phi_{1}-\Delta \phi_{2}\right)=0$, leading to two pulse trains that have nearly 


\section{REPOR T S}

identical dynamical evolution.

The comb can be characterized by two variables; $f_{\text {rep }}=v_{\mathrm{g}} / l_{\mathrm{c}}$ and $\delta=v_{0}\left(1-v_{\mathrm{g}} / \nu_{\mathrm{p}}\right)$, where $v_{0}$ is the laser carrier frequency and $l_{\mathrm{c}}$ the cavity length. One obvious choice of laboratory control variables is $l_{\mathrm{c}}$, which controls $f_{\text {rep }}$ without any influence on $\delta$. Another control mechanism would be to vary either the pump laser power, which influences both $v_{\mathrm{g}}$ and $v_{\mathrm{p}}$, or to vary the angle of a swivel mirror in the dispersed end of the laser cavity to control $v_{\mathrm{g}}$. Here, we use a fast servo loop acting on $l_{\mathrm{c}}$ to stabilize $f_{\text {rep}}$, whereas the offset frequency $\delta$ is controlled using the swivel mirror [Supplemental fig. 1 (23)]. Fluctuations of $\delta$ develop on a slower time scale compared with that of $f_{\text {rep }}$, and therefore a correspondingly slower servo loop is sufficient for stabilization of $\delta$.

The two independent mode-locked Ti:sapphire lasers (24) each operate at a $100 \mathrm{MHz}$ repetition rate, with one centered at $760 \mathrm{~nm}$ and the other at $810 \mathrm{~nm}$. The bandwidth of each laser corresponds to a sub-20 fs transform limited pulse. Our synchronization scheme employs two phase-locked loops working at different time resolutions [Supplemental fig. 1 (23)]. The first loop operates at the fundamental repetition frequency to provide full dynamic range of timing offset, whereas the second one operates at the 80 th harmonic of $f_{\text {rep }}(8 \mathrm{GHz})$ to provide enhanced phase stability. Using this approach, we have demonstrated the timing jitter between the two separate pulse trains to be $<5 \mathrm{fs}$, at a bandwidth of $160 \mathrm{~Hz}$, observed over several minutes (25). This result represents a nearly 100-fold improvement over previous studies in synchronizing separate mode-locked
Ti:sapphire lasers (26). The flexibility of this synchronization system is demonstrated by locking two independent lasers working at different commensurable repetition frequencies.

When the two lasers are well synchronized, the heterodyne beat between the two corresponding sets of combs can be recovered with a signal-to-noise ratio of $60 \mathrm{~dB}$ in a $100-\mathrm{kHz}$ bandwidth. Phase shift in the synchronization lock loop is adjusted to have the two pulses optimally overlapped temporally at the heterodyne detector to produce the maximum beat signal. The beat detection effectively measures the difference $\left(\delta_{1}-\delta_{2}\right)$ in the carrier-envelope offset frequency $\delta$ between the two fs combs. Hundreds of comb pairs contribute to the heterodyne beat signal, and its amplitude is coherently enhanced when the synchronization is effective. By stabilizing $\left(\delta_{1}-\delta_{2}\right)$ to a mean value of $0 \mathrm{~Hz}$, the carrier-envelope phase slip per pulse of one laser will be accurately matched by the second laser. Locking of this beat frequency to $0 \mathrm{~Hz}$ is implemented using an acoustooptic modulator (AOM). One laser beam passes through the AOM and is offset by the drive frequency of the AOM. This avoids the need to process the beat signal in the troublesome frequency range around $0 \mathrm{~Hz}$. The beat is then phase-locked to the drive frequency of the AOM, effectively removing the AOM frequency from the beat.

The carrier frequency beat signal between the two lasers is recorded under phase-locked conditions (Fig. 2A), with a standard deviation of $0.15 \mathrm{~Hz}$ at a $1-\mathrm{s}$ averaging time. In contrast, without phase locking, the beat frequency between the two synchronized lasers
Fig. 1. The principle behind the synchronization and phase locking of two independent femtosecond lasers. (A) Pulse representation in the time domain; (B) Femtosecond comb representation in the frequency domain. $f_{\text {rep }}$ represents the laser repetition frequency (comb spacing) whereas $\delta$ denotes the offset frequency in the comb due to the carrier-envelope phase slip $\Delta \phi$.

\section{A Time domain}

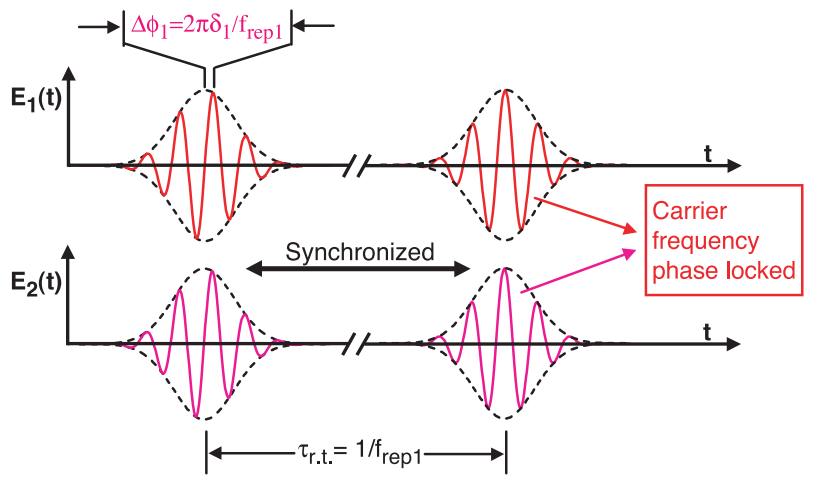

B Frequency domain

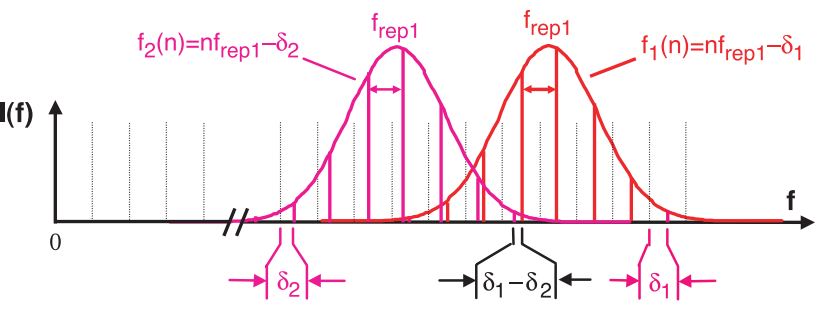

has a standard deviation of a few MHz. To further explore the issue of the beat frequency noise versus characteristic time scales, we employ an Allan variance analysis (27), which allows study of noise process in the time domain by comparing adjacent measurements segmented within certain time windows. In this first-difference calculation, processes can be separated and isolated on the basis of their time scales, permitting the time scales at which fluctuations are the largest to be identified. From the time record of the timing jitter noise, we determined the corresponding Allan variance (red diamonds, Fig. 2B). Another fast frequency counter measures Allan variance directly on the instrument (blue circles, Fig. 2B). The result shows that the frequency variance falls off at a rate of $1 / \tau$, where $\tau$ is the averaging time. This is characteristic of phase noise in our widerange phase-locked system.

Although this frequency counting method is convincing, a direct time-domain analysis of the coherence between the two fs lasers can be equally effective [Supplemental figure 2 (23)]. Spectral interferometry is a sensitive technique for measuring frequency-domain phase difference between two pulses, and it has been applied to the characterization of weak signal pulses with respect to stronger reference pulses (28) and to pulse-shaping studies. We combine the pulses from the two lasers on a beam splitter and direct the two pulses collinearly into a spectrometer consisting of a grating and a charge-coupled device array. The spectrum of the sum of the two pulses would produce the phase difference between the two pulses, and a preset delay $\tau$ between the two pulses would yield fringes in the combined spectrum, if coherence between the two pulse trains persists over the measurement time period. Typical

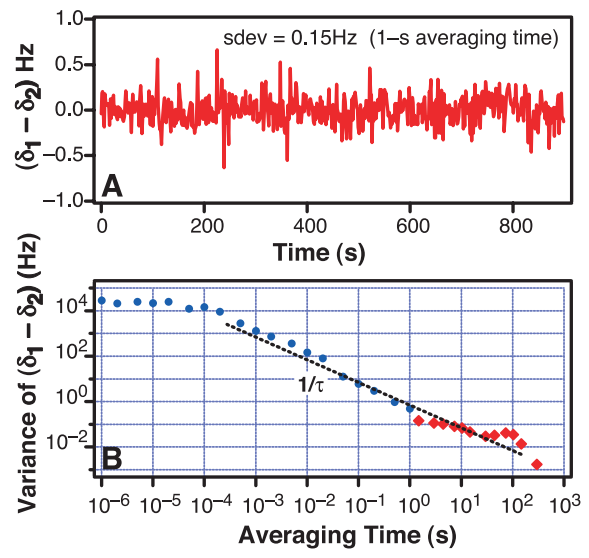

Fig. 2. (A and B) Time record of the carrier beat frequency between the two femtosecond lasers when phase-locked. The associated Allan variance (in red squares) is also shown to reflect the noise process at different averaging time scales. The Allan variance at shorter time scales (in blue circles) is measured with an independent frequency counter. 


\section{REPOR T S}

spectral interferometry data (Fig. 3A) when the spectra of the two lasers are overlapped are shown. The black and green curves represent the two individual pulse spectra, obtained by blocking one beam and measuring the spectrum of the other. When both pulses are sent into the spectrometer, the resulting sum spectrum is shown in blue (without phase locking between the two lasers) and in red (with phase locking). Whereas the spectrum is smooth in the nonphase locked case, under phase-locked conditions, interference fringes are produced that reveal excellent phase coherence between the two pulses within the observation time, chosen to be $20 \mu \mathrm{s}$ in this case. Timing jitter at this short time scale is measured to be $\leq 1 \mathrm{fs}$. When the gate time is increased, we observe a decreased contrast in the interference fringes. However, the fringe pattern is still visible even at relatively long observation times of $20 \mathrm{~ms}$.

Cross-correlation measurements of the two field amplitudes is another powerful technique to demonstrate mutual phase coherence (29). The interference term in a cross-correlation measurement is a convolution integral of the two field amplitudes, and fringes are obtained by scanning the delay between the two pulses. The second-order field cross-correlation is obtained when the coherent sum of the two optical fields passes

Fig. 3. Coherence measurement between the two phase-locked femtosecond lasers. (A) Spectral interferometry: the black and green curves represent the individual laser's spectrum; the blue curve shows the combined spectrum of the two lasers when they are not phase-locked; the red curve shows clear interference fringes between the two laser spectra when they are phase-locked. (B) Second-order field crosscorrelation: the blue curve indicates the lack of coherence between the two optical fields from the two phase-independent (but synchronized) lasers; the red curve shows cross-cor-
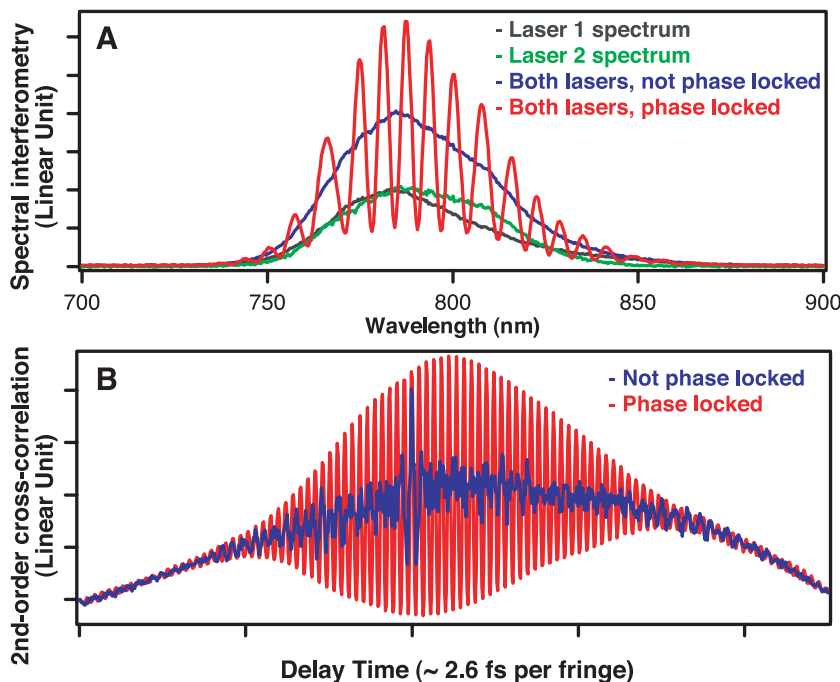

relation fringes between the two phase-locked lasers.

Fig. 4. Second-order autocorrelation measurement of the combined pulse. Second-order autocorrelation data of the two individual lasers are shown in (A) and (B). In (C), the spectral interferometry data indicate the spectra of the two lasers are separated, with interference fringes clearly present in the overlap region of the two spectra. The pulse trains from the two lasers are combined and sent to a collinear autocorrelator. Data in (D) represents the case when the two lasers are not synchronized. The two fields are in phase only briefly, resulting in a random sharp spike in the
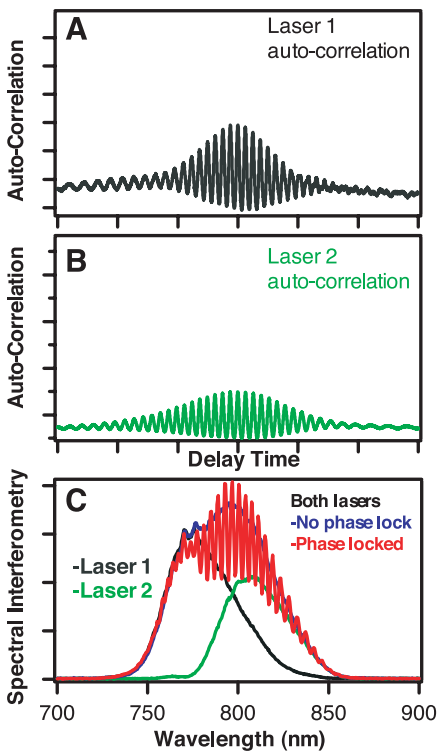
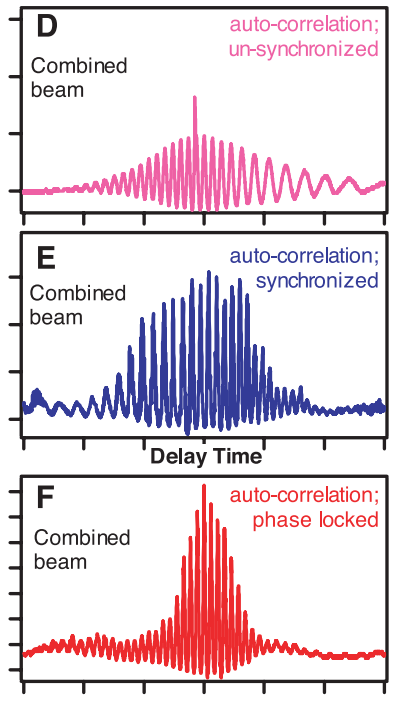

Delay Time ( 2.6 fs per fringe) autocorrelation curve.

Data in (E) are obtained when the two lasers are synchronized but not phase-locked. The increase of the autocorrelation amplitude is about 2.7 times over the previous unsynchronized case. When the two lasers are both synchronized and phase-locked, the second-order autocorrelation data in (F) show the pulse width is narrowed and pulse amplitude increased by $>20 \%$. Notice the large difference in noise between the phase-locked and unlocked autocorrelation curves. through a second harmonic crystal before entering the photodetector. Again, we compare the phase locked (red curve) and unlocked (blue curve) results in Fig. 3B. Each scan takes about $20 \mu \mathrm{s}$, and the data shown here are averaged results of eight scans. The fringe contrast of the averaged scans is reduced by only $20 \%$ from a single scan when phase lock is active. Without phase locking, the fringe contrast quickly disappears after averaging only a few sweeps.

To demonstrate the "coherently synthesized" aspect of the combined pulse, a second-order autocorrelation measurement of the combined pulse is carried out. For this measurement, the two pulse trains were maximally overlapped in the time domain before the autocorrelator. One arm of the autocorrelator (Michelson interferometer) was scanned to collect the autocorrelation data. The second-order autocorrelation curves of each individual laser are shown (Fig. 4, A and B, respectively). The spectrum of one laser is centered around $760 \mathrm{~nm}$, whereas the other one around $810 \mathrm{~nm}$. Under phase lock, the spectral interferometry measurement displays clear interference fringes within the overlapping spectra (Fig. 4C). An interesting autocorrelation measurement is shown when the two lasers are not even synchronized (Fig. 4D). We obtain an autocorrelation of a single laser pulse, with a sharp spike appearing in the data at a random position. The spike appears because, at that particular instant, the pulses overlapped in time and the two electric fields came into phase and coherently added together. The time scale of this random interference is related to the offset frequency between the two repetition rates and is usually less than a few ns. When the two lasers are synchronized but not phase-locked, the resulting autocorrelation measurement indicates an increased signal amplitude compared to the unsynchronized case, typically by a factor of 2.7 (Fig. 4E). However, as expected, this signal displays considerable random phase noise within the autocorrelation interference fringes. When the two lasers are phase-locked, the autocorrelation reveals a clean pulse that is often shorter in apparent duration and larger in amplitude (Fig. 4F). This result is statistically significant because this pulse shortening is observed in more than $40 \%$ of the total data scans. Note, however, that in this work we did not attempt to recompress the light pulses outside the laser cavities to very short duration, and the pulses are dispersively broadened to 50 to $70 \mathrm{fs}$ in duration. The width of the central fringe pattern in an interferometric autocorrelation is more characteristic of the overall bandwidth of the pulse than of the pulse duration, and can result in a trace that appears deceptively short when the overall timespan of the scan is short. However, the data clearly show the difference between a coherently phase-locked pulse train (Fig. 4F) and one with a complex and random frequency substructure (Fig. 4E). Averaging of hundreds 
of the autocorrelation scans also consistently shows an amplitude enhancement by more than $20 \%$ when the phase lock is activated.

An important issue will be to demonstrate control over the phase profile across the entire synthesized spectrum, namely pulse shaping. For example, a flat spectral phase profile is a prerequisite for generating an ultrashort pulse while arbitrary shape is needed for coherent control applications. Using the current control scheme, the carrier-envelope slip phases of the two lasers track each other $\left(\Delta \phi_{1}=\Delta \phi_{2}\right)$. There remains, of course, a static phase difference between the two lasers, namely $\left(\phi_{1}-\phi_{2}\right)$. However, this static phase can be controlled through an appropriate phase offset introduced in the carrier heterodyne beat detection, for example, through phase shift of the radio frequency signal driving the AOM. This phase-compensated spectrum can then be used in a pulseshaping device to generate the desired pulse waveform.

\section{References and Notes}

1. M. H. Anderson, J. R. Ensher, M. R. Matthews, C. E. Wieman, E. A. Cornell, Science 269, 198 (1995).

2. C. A. Sackett et al., Nature 404, 256 (2000).

3. R. S. Judson, H. Rabitz, Phys. Rev. Lett. 68, 1500 (1992).

4. D. E. Spence, P. N. Kean, W. Sibbett, Opt. Lett. 16, 42 (1991).

5. J. P. Zhou et al., Opt. Lett. 19, 1149 (1994).

6. R. Ell et al., Opt. Lett. 26, 373 (2001).

7. R. R. Alfano, The Supercontinuum Laser Source (Springer-Verlag, New York, 1989).

8. J. K. Ranka, R. S. Windeler, A. J. Stentz, Opt. Lett. 25, 25 (2000).

9. P. B. Corkum, N. H. Burnett, M. Y. Ivanov, Opt. Lett. 19, 1870 (1994).

10. A. E. Kaplan, Phys. Rev. Lett. 73, 1243 (1994); A. V. Sokolov, D. R. Walker, D. D. Yavuz, G. Y. Yin, S. E. Harris, Phys. Rev. Lett. 85, 562 (2000).

11. R. A. Kaindl et al., J. Opt. Soc. Am. B 17, 2086 (2000).

12. R. W. Schoenlein et al., Science 274, 236 (1996).

13. R. L. Byer, J. Mod. Opt., in press.

14. J. Ye, J. L. Hall, Opt. Lett. 24, 1838 (1999).

15. T. Udem, J. Reichert, R. Holzwarth, T. W. Hänsch, Opt. Lett. 24, 881 (1999).

16. _ Phys. Rev. Lett. 82, 3568 (1999).

17. S. A. Diddams et al., Phys. Rev. Lett. 84, 5102 (2000).

18. J. Ye, J. L. Hall, S. A. Diddams, Opt. Lett. 25, 1675 (2000).

19. L. Hollberg et al., IEEE J. Quantum Electron., in press. 20. D. J. Jones et al., Science 288, 635 (2000).

21. A. Apolonski et al., Phys. Rev. Lett. 85, 740 (2000).

22. H. R. Telle et al., Appl. Phys. B 69, 327 (1999).

23. Supplemental material is available at Science Online at www.sciencemag.org/cgi/content/full/293/5533/ 1286/DC1

24. M. T. Asaki et al., Opt. Lett. 18, 977 (1993).

25. L. S. Ma, R. K. Shelton, H. Kapteyn, M. Murnane, J. Ye, Phys. Rev. A, 64, 021802(R) (2001).

26. S. A. Crooker, F. D. Betz, J. Levy, D. D. Awschalom, Rev. Sci. Instrum. 67, 2068 (1996).

27. D. W. Allan, Proc. IEEE 54, 221 (1966).

28. D. N. Fittinghoff et al., Opt. Lett. 21, 884 (1996).

29. J.-C. Diels, W. Rudolph, Ultrashort Laser Pulse Phenomena: Fundamentals, Techniques, and Applications on a Femtosecond Time Scale (Academic Press, San Diego, CA, 1996).

30. We are indebted to D. Anderson for the loan of essential equipment and S. T. Cundiff for critical comments. We also thank R. Bartels, T. Weinacht, and S. Backus for useful discussions. The work at JILA is supported by NIST, NASA, NSF, and the Research Corporation.

19 April 2001; accepted 4 July 2001

\title{
Nanowire Nanosensors for Highly Sensitive and Selective Detection of Biological and Chemical Species
}

\begin{abstract}
Yi Cui, ${ }^{1 *}$ Qingqiao Wei, ${ }^{1 *}$ Hongkun Park, ${ }^{1}$ Charles M. Lieber ${ }^{1,2} \dagger$
Boron-doped silicon nanowires (SiNWs) were used to create highly sensitive, real-time electrically based sensors for biological and chemical species. Amineand oxide-functionalized SiNWs exhibit $\mathrm{pH}$-dependent conductance that was linear over a large dynamic range and could be understood in terms of the change in surface charge during protonation and deprotonation. Biotin-modified SiNWs were used to detect streptavidin down to at least a picomolar concentration range. In addition, antigen-functionalized SiNWs show reversible antibody binding and concentration-dependent detection in real time. Lastly, detection of the reversible binding of the metabolic indicator $\mathrm{Ca}^{2+}$ was demonstrated. The small size and capability of these semiconductor nanowires for sensitive, label-free, real-time detection of a wide range of chemical and biological species could be exploited in array-based screening and in vivo diagnostics.
\end{abstract}

Planar semiconductors can serve as the basis for chemical and biological sensors in which detection can be monitored electrically and/or optically (1-4). For example, a planar field effect transistor (FET) can be configured as a sensor by modifying the gate oxide (without gate electrode) with molecular receptors or a selective membrane for the analyte of interest; binding of a charged species then results in depletion or accumulation of carriers within the transistor structure $(1,2)$. An attractive feature of such chemically sensitive FETs is that binding can be monitored by a direct change in conductance or related electrical property, although the sensitivity and potential for integration are limited.

The physical properties limiting sensor devices fabricated in planar semiconductors can be readily overcome by exploiting nanoscale FETs (5-9). First, binding to the surface of a nanowire (NW) or nanotube (NT) can lead to depletion or accumulation of carriers in the "bulk" of the nanometer diameter structure (versus only the surface region of a planar device) and increase sensitivity to the point that single-molecule detection is possible. Second, the small size of NW and NT building blocks and recent advances in assembly $(9,10)$ suggest that dense arrays of sensors could be prepared. Indeed, NT FETs were shown recently by Dai and co-workers to function as gas sensors (11). Calculations

1Department of Chemistry and Chemical Biology, Harvard University, Cambridge, MA 02138, USA. ²Division of Engineering and Applied Sciences, Harvard University, Cambridge, MA 02138 USA.

*These authors contributed equally to the work. †To whom correspondence should be addressed. Email: cml@cmliris.harvard.edu suggested that direct binding of electronwithdrawing $\mathrm{NO}_{2}$ or electron-donating $\mathrm{NH}_{3}$ gas molecules to the NT surface chemically gated these devices. However, several properties of NTs could also limit their development as nanosensors, including the following: (i) existing synthetic methods produce mixtures of metallic and semiconducting NTs, which make systematic studies difficult because metallic "devices" will not function as expected, and (ii) flexible methods for the modification of NT surfaces, which are required to prepare interfaces selective for binding a wide range of analytes, are not well established.

Nanowires of semiconductors such as $\mathrm{Si}$ do not have these limitations, as they are always semiconducting, and the dopant type and concentration can be controlled (7-9), which enables the sensistivity to be tuned in the absence of an external gate. In addition, it should be possible to exploit the massive knowledge that exists for the chemical modification of oxide surfaces, for example, from studies of silica (12) and planar chemical and biological sensors $(4,13)$, to create semiconductor NWs modified with receptors for many applications. Here we demonstrate the potential of NW nanosensors with direct, highly sensitive real-time detection of chemical and biological species in aqueous solution.

The underlying concept of our experiments is illustrated first for the case of a $\mathrm{pH}$ nanosensor (Fig. 1A). Here a silicon NW (SiNW) solid state FET, whose conductance is modulated by an applied gate, is transformed into a nanosensor by modifying the silicon oxide surface with 3-aminopropyltriethoxysilane (APTES) to provide a surface 
If you wish to distribute this article to others, you can order high-quality copies for your colleagues, clients, or customers by clicking here.

Permission to republish or repurpose articles or portions of articles can be obtained by following the guidelines here.

The following resources related to this article are available online at www.sciencemag.org (this information is current as of July 17, 2015 ):

Updated information and services, including high-resolution figures, can be found in the online version of this article at:

http://www.sciencemag.org/content/293/5533/1286.full.html

Supporting Online Material can be found at:

http://www.sciencemag.org/content/suppl/2001/08/16/293.5533.1286.DC1.html

A list of selected additional articles on the Science Web sites related to this article can be found at:

http://www.sciencemag.org/content/293/5533/1286.full.html\#related

This article cites 24 articles, 3 of which can be accessed free:

http://www.sciencemag.org/content/293/5533/1286.full.html\#ref-list-1

This article has been cited by 134 article(s) on the ISI Web of Science

This article has been cited by 6 articles hosted by HighWire Press; see:

http://www.sciencemag.org/content/293/5533/1286.full.html\#related-urls

This article appears in the following subject collections:

Physics, Applied

http://www.sciencemag.org/cgi/collection/app_physics 